\title{
Big data y Relaciones Públicas. Una revisión bibliográfica del estado de la cuestión
}

\section{Big Data and Public Relations. A literature review}

\author{
Pereira Villazón, T., Portilla Manjón, I. y Rodríguez Salcedo, N. ${ }^{1}$ \\ Recibido: 26-09-2018 - Aceptado: 30-01-2019 \\ DOI: https://doi.org/10.26441/RC18.1-2019-A8
}

RESUMEN: La creciente actividad generada en las plataformas digitales de comunicación está suscitando nuevas formas de relación entre las organizaciones y sus públicos de interés. Esto ha desencadenado, a su vez, la producción de una gran cantidad de información, una explosión de datos conocida como Big Data.

En este nuevo contexto, surge la necesidad de estudiar cómo se afronta en Relaciones Públicas el manejo de esta ingente cantidad de información, que se genera de manera constante, a gran velocidad y que exige una gestión incluso en tiempo real.

Este trabajo realiza una revisión bibliográfica del estado actual de la investigación sobre la gestión de Big Data en Relaciones Públicas. El resultado del análisis es un total de 41 trabajos. A través del análisis de contenido, se constata que las actividades de comunicación interna, relaciones con los medios, comunicación de crisis e issues management son las más vinculadas a la gestión de datos masivos.

Palabras Clave: relaciones públicas; Big Data; gestión de datos y comunicación.

ABSTRACT: The growing activity generated in digital communication platforms is raising new forms of relationship between organizations and their publics of interest. This has triggered, in turn, the production of a large amount of information, an explosion of data known as Big Data.

In this new context, there is a need to study how the management of this huge amount of information faced in public relations, which is constantly generated at high speed and which requires even realtime management.

In this work, we present a bibliographic review of the current state of research on the management of Big Data in Public Relations. The results showed a total 41 works. A content analysis of these works found that the activities of internal communications, media relations, crisis communications and issues management are the most related to massive data management.

Keywords: public relations; Big Data; data management and Communication.

1 Tatiana Pereira Villazón es Master en Comunicación empresarial, Business/Corporate Communication por la Universitat de Barcelona IL3 y Doctoranda en Comunicación en la Universidad de Navarra. tpereira@alumni. unav.es, https://orcid.org/0000-0003-4238-5300

Idoia Portilla Manjón es Doctora en Ciencias Económicas y Empresariales por la Universidad de Navarra y Profesora de Análisis de información e Investigación de Mercados y Estadística en la Facultad de Comunicación de la misma universidad. iportilla@unav.es, https://orcid.org/0000-0002-2504-868X

Natalia Rodríguez Salcedo es Doctora en Comunicación por la Universidad de Navarra y Profesora de Introducción a las Relaciones Públicas, Historia de las Relaciones Públicas y Gestión de Asuntos Públicos en la Facultad de Comunicación de la misma universidad.nrodriguez@unav.es, http://orcid.org/0000-0003-0968-0447 


\section{Introducción}

La digitalización de la comunicación y el constante crecimiento de las nuevas tecnologías han planteado nuevos retos y oportunidades en el campo de las Relaciones Públicas. Estos cambios han situado a la disciplina en la necesidad de adaptarse a los desafíos y oportunidades planteados por las tecnologías de información y comunicación.

La creciente conectividad, las múltiples pantallas y las plataformas de comunicación han traído consigo nuevas formas de relación entre la organización y sus públicos que, cada vez más, producen y consumen contenidos en las redes y plataformas sociales. Todo ello ha desencadenado que las organizaciones están tratando con una gran cantidad de datos (Davenport, Barth \& Bean, 2012).

Como señala Martin (2007), estamos en la "era de la sociedad del aprendizaje" que implica nuevas formas de gestionar datos en las actividades de Relaciones Públicas. Por tanto, la digitalización viene de la mano de cambios en la gestión de datos, en particular el Big Data (Klewes, Popp \& Rost-Hein, 2017).

Dicha gestión de datos por parte de las organizaciones está directamente vinculada con la teoría de las relaciones públicas, Como apunta Benítez-Eyzaguirre (2016) el fin último el proceso de comunicación y de alianzas es establecer un diálogo entre organizaciones y públicos, lo que conlleva la administración y gestión de datos por parte de la disciplina de las Relaciones Públicas.

La gestión de datos no es nueva en el ámbito de la comunicación, ni en el campo de las Relaciones Públicas. De hecho, la reputación corporativa es un área que ha venido manejando mucha información. Según Orozco y Ferre (2012), resulta fundamental conocer numéricamente los efectos sobre la reputación de las estrategias de comunicación y el diálogo con los stakeholders de las organizaciones, para que los datos permitan tomar decisiones acertadas dentro de las organizaciones.

Los datos también han sido relevantes en la gestión de crisis y en la relación con los medios. En estos casos han sido necesarios datos tan simples como el número de personas que asisten a un evento, o tan complejos como la credibilidad de la organización basada en un esquema de medición (Stacks, 2017).

En el entorno de la comunicación actual, la globalización y las redes sociales acrecientan la necesidad de nuevas prácticas en relaciones públicas (Wichels, 2014). Dichas prácticas permitirán la rápida y oportuna gestión de datos masivos, así como también, la recopilación de datos únicos de usuarios con el fin de obtener una comunicación efectiva con ellos.

En esta línea, el propósito de este trabajo es realizar una revisión bibliográfica, a través del análisis de contenido, del estado de la cuestión sobre cómo se trata el tema del Big Data vinculado a las Relaciones Públicas en las publicaciones académicas. En concreto, se trata de comprobar en qué medida ha llegado al ámbito en cuanto a número de trabajos, idioma de publicación, la fecha y tipo de publicación (artículos, tesis y capítulos de libro, entre otros). 
En segundo lugar, se describirán las actividades de Relaciones Públicas vinculadas con el uso del Big data a las que se presta atención en las publicaciones. Finalmente se sintetizarán los temas de estudio de interés por parte de los investigadores relacionados con Big data y Relaciones Públicas.

\section{Aproximación al concepto Big Data}

Big Data se conceptualiza habitualmente con tres Vs: volumen, velocidad y variedad. Es el marco común para describir a los datos masivos (Chen, Chiang \& Storey, 2012; Gandomi \& Haider 2015). Más explícitamente, la consultora Gartner (2012) define "Big Data como un gran volumen, alta velocidad y / o gran variedad de activos de información que demandan formas rentables e innovadoras de procesamiento de la información que permiten un mejor conocimiento, toma de decisiones y automatización de procesos".

Volumen y velocidad son dos de las características que más destacan en las definiciones (Yanik \& Göztaş, 2015). Pero, como apunta Swinson y Woliansky (2014), los datos masivos se caracterizan por ser "conjuntos muy grandes de datos a menudo no estructurados". No se trata de bases de datos de filas y columnas (estructuradas), sino en nuevas formas que precisan nuevos sistemas de trabajo, y que recogen una gran variedad de formatos de información, desde datos numéricos y de texto hasta imágenes o vídeos.

Pero estas tres Vs iniciales (volumen, velocidad y variedad) se acompañan en algunos casos de nuevas Vs. Según
Gandomi y Haider (2015), una es la "veracidad" (acuñada por IBM) que representa la fiabilidad de las fuentes de datos y el "grado en el cual se puede confiar en ellos, dada la confiabilidad de su fuente" (Wang, Xu, Fujita \& Liu, 2016: 4).

Otra nueva $\mathrm{V}$ es la característica del "valor", que implica que los datos deben tener significado para poder ayudar a las organizaciones en la toma de decisiones. Se puede vincular al uso del término "Smart Data", primando la calidad del dato sobre su cantidad, de modo que los datos sean realmente un activo para las organizaciones (Bustamante \& Guillén, 2017).

Por otra parte, Boyd y Crawford (2012) definen Big data como "la capacidad para recopilar, analizar, vincular y comparar grandes conjuntos de datos" (p.663). Es decir, se centran más en los procesos que exigen la gestión de los datos masivos, en lugar de en las características de estos últimos.

Más aún, podemos encontrar diferentes usos del término Big Data por sector, objeto de estudio en el que se trabaje, orientación del investigador y/o perspectivas que se aborde, desde la orientada a productos, procesos, a la cognición y perspectiva del movimiento social (Wang et al, 2016).

Sin embargo, esta falta de unidad en la definición no es un problema. Más aún, esta ambigüedad "puede haber ayudado a Big Data a abrirse camino en una variedad de espacios académicos" (Shahin, 2016: 977). La necesidad de elaborar estrategias, recopilar y analizar datos es fundamental para las 
organizaciones (Weinner \& Kochhar, 2016a), también en el área de comunicación.

\section{Relaciones Públicas y Big Data}

La disciplina de las Relaciones Públicas es multifacética (Xifra, 2006) y está en constante evolución debido a su gran adaptabilidad a los cambios, a los retos que se generan dentro del ambiente organizacional y su interrelación con distintas disciplinas con las que se relaciona en el mundo empresarial.

A raíz de dicha circunstancia "la elección de una u otra expresión lleva con frecuencia implícita una opción teóricometodológica y una delimitación del campo objeto de estudio, que necesariamente incide en la perspectiva y fines de la indagación científica” (Xifra, 201: 20).

Es por ello que las actividades incluidas en esta disciplina pueden variar de unos autores a otros. Si consideramos la clasificación de Broom y Sha (2013), en relaciones públicas se atienden cuestiones de comunicación interna, relaciones con los medios, lobbying y/o gestión de asuntos públicos, issues management, publicity, comunicación o manejo de crisis, relaciones con los inversores y comunicación de desarrollo

Independientemente de las actividades que se consideren, la irrupción de Big data tanto en el campo de la comunicación (Shain, 2016), como en diferentes áreas del conocimiento, ha generado nuevas formas de comprender la concepción de trabajo. (Puyol, 2015).

Saber gestionar y analizar Big Data es invertir en el futuro a través de la innovación. Como bien señala Puyol
(2015), "el siglo XXI será el siglo de los datos, así como el siglo XX fue declarado el siglo del petróleo" (p. 197).

Sin embargo, la irrupción de Big Data no debe verse como una amenaza, sino como una oportunidad:

"Para superar los enfoques tradicionales de comunicación, para crear mejores objetivos, posicionamiento más estratégico y público objetivo, y descubrir más oportunidades para cuantificar y mejorar la contribución de las RRPP al éxito empresarial y su influencia en la línea de fondo" (Weiner \& Kochhar, 2016: 15).

La superación de enfoques tradicionales conlleva la administración y gestión oportuna de información que representa una gran cantidad de datos. Las previsiones de crecimiento global estiman que la cantidad de datos digitales formados en un año, es mayor que la cantidad generada en los últimos 50 años (Yanik \& Göztaş, 2015). Se estima que los datos producidos a nivel mundial aumentarán un $800 \%$ en los próximos cinco años, según los investigadores de mercado de la consultora Gartner (Heitmann, 2016).

La aplicación de Big data a la gestión estratégica de la comunicación ofrece nuevas oportunidades, además de plantear cuestiones acerca de la interpretación de los datos masivos (Holtzhausen, 2016). Uno de los principales usos de Big Data se presenta en la comunicación con audiencias específicas, creando así un vínculo directo entre Big Data y comunicación.

Tanto los profesionales como los académicos de Relaciones Públicas deben 
afrontar el reto y comprender la nueva cultura empresarial y económica en la cual la conectividad obliga a rediseñar estrategias de comunicación corporativa (Benítez-Eyzaguirre, 2016).

Adquirir y utilizar datos de diversas fuentes para tomar decisiones y ofrecer mejores productos en el campo las relaciones públicas y la comunicación estratégica es vital, aunque la discusión sobre el tema es muy reciente (Weiner \& Kochar, 2016).

Resulta imprescindible comprender la Big Data no solo como datos que el profesional puede analizar sino como una estrategia de datos. Como tal, las organizaciones deberían reorganizarse según nuevos estándares para realizar una gestión adecuada de los mismos (Yanik \& Göztaş, 2015).

Las empresas deben adaptarse e ir evolucionando a ritmo de las nuevas tecnologías y los profesionales de Relaciones Públicas pueden "ayudar a asegurar el nuevo enfoque empresarial de un alto nivel de conocimiento y aceptación rápidamente" (Klewes, et al, 2017: 33).

El Big Data será esencial para el desempeño de las actividades de Relaciones Públicas porque la utilización de datos y métricas pueden resultar eficaces cuando se aplican como herramienta que no solo analizan patrones, sino que también pueden proporcionar la probabilidad predictiva necesaria para un evento (George, Haas \& Pentland, 2014), o en la gestión de una crisis (Wichels, 2014).

El análisis del Big Data puede ser efectivo para examinar la opinión pú- blica y también en el desarrollo de estrategias de relaciones públicas de manera efectiva con las partes interesadas (Choi, 2015). El vínculo entre la función de gestión de Relaciones Públicas y el potencial del uso de análisis del Big Data es imprescindible para mejorar la comprensión y la comunicación entre las organizaciones y sus públicos (Holtzhausen, 2016; Weiner \& Kochhar, 2016; Yanki \& Göztaş, 2015).

\section{Metodología}

\subsection{Objetivos y Método}

Vista la relevancia de la aplicación del Big Data en Relaciones Públicas, el estudio se centra en el desarrollo del tema en el área académica. Concretamente, en los siguientes objetivos:

- Describir el estado de la investigación académica sobre Big Data y Relaciones Públicas. Se trata de comprobar en qué medida ha llegado al ámbito en cuanto a: 1) número de trabajos 2) idioma de la publicación (inglés, español); 3) fecha de publicación; 4) tipo de publicación (artículos, tesis, trabajos fin de grado o máster, libros, capítulos de libros, o informes/estudios). Se tratará además de observar qué revistas y/o libros han destacado en el tratamiento de estos temas.

- Describir las actividades de Relaciones Públicas vinculadas con el uso del Big data a las que se presta atención en las publicaciones. Siguiendo la clasificación de Broom y Sha (2013), se consideran: comunicación interna, publicity, relación 
con los medios, lobbying y/o gestión de asuntos públicos, issues management, comunicación de crisis, relaciones con los inversores y comunicación para el desarrollo.

- Conocer qué áreas y temas han sido tratados y, teniendo en cuenta los resultados, detectar cuales pueden tener mayor desarrollo.

Para lograr este propósito se realiza un análisis de contenido. Este método permite estudiar y analizar la comunicación de manera sistemática, objetiva y cuantitativa con el fin de medir variables (Kerlinger \& Lee, 2000).

La codificación de las actividades de relaciones públicas, se realizó describiendo cada una de ellas según la clasificación de Broom y Sha (2013). Dicha conceptualización fue necesaria para definir los límites de las categorías y también para comprender cada una de las actividades, como apuntan Wimmer y Dominick (2011).

\subsection{Muestra y unidades de análisis}

La búsqueda y selección de publicaciones se realizó con dos metabuscadores. En primer lugar, se utilizó la herramienta de búsqueda UNIKA, desarrollada por la Biblioteca de la Universidad de Navarra (http://www.unav.edu/web/biblioteca). Esta herramienta permite buscar en el catálogo conformado por libros, revistas impresas, $C D$ Roms, la base de datos $\mathrm{SABIO}$, portales de revistas y base de datos por materia EBSCO, Web of Science, Dialnet, etc, así como DADUN el repositorio digital institucional de la Universidad.

En segundo lugar, para complementar la búsqueda realizada con UNIKA, se utilizó el metabuscador Google Académico (https://scholar.google.es/). Se trata de una herramienta de búsqueda de Google especializada en la búsqueda de trabajos y publicaciones científico-académicas.

La selección de artículos y publicaciones se realizó, en primer lugar, utilizando combinaciones de términos clave. Concretamente se utilizaron "Relaciones públicas y Big Data", "Gestión de datos y relaciones públicas", "Big data y comunicación", "gestión de datos y comunicación", "gestión de información y "relaciones públicas", tanto en castellano como en inglés.

Dichos términos debían localizarse en el título, resumen y/o palabra clave de las publicaciones, con el fin de asegurarnos que los trabajos abordarán el tema. No se limitó la búsqueda por fecha, ni por tipo de publicación.

Una vez obtenida la primera selección de trabajos, se leían para corroborar que trataban el tema. Tras la lectura, fue necesario eliminar trabajos en los que figuraban las palabras Relaciones Públicas o comunicación como profesión del autor o área en que trabaja, o incluían estos términos, pero no se podían considerar que tratasen el tema del Big Data vinculado a las Relaciones Públicas. De este modo se seleccionaron solo las publicaciones que abordaban, en una parte o en todo el texto, el objeto de estudio.

La búsqueda se realizó durante el mes de diciembre de 2017. El resultado final arrojó una muestra de 41 publicaciones, de las cuales 32 corresponden a artículos, tres capítulos en tres libros, dos trabajos 
fin de grado y uno de master, una tesis de doctorado y dos informes/estudios. Dado el reducido número, se mantuvieron todas ellas en el estudio, de modo que mostrasen todas las líneas que trabajan el Big Data en el área de Relaciones Públicas. Por tanto, cada publicación hallada se convirtió en una unidad de análisis para el análisis de contenido.

\subsection{Clasificación de unidades de análisis}

Los 41 trabajos analizados se clasificaron inicialmente atendiendo a la fe- cha de publicación (año), al tipo y fuente de publicación (Ver Tabla 1). También se consideró el idioma en que se publicaban (inglés o castellano) y en qué revistas, libros o universidades. Tras la lectura completa del trabajo, el análisis se completa con una clasificación atendiendo a los siguientes aspectos:

- Actividad de las relaciones públicas según la clasificación de los autores Broom y Sha (2013).

- Temáticas concretas tratadas por los autores.

Tabla 1. Clasificación de publicaciones

\begin{tabular}{|c|l|c|}
\hline Año & \multicolumn{1}{|c|}{ Fuente de Publicación } & $\begin{array}{c}\text { Tipo de } \\
\text { publicación y no }\end{array}$ \\
\hline 2005 & Scire Representación y organización del conocimiento & Artículo \\
\hline 2013 & Libro: Share This Too: More Social Media Solution for PR Professionals & 1 Capitulo \\
\hline & Revista: Public Relations Tactics (Public Relations of America) & Artículo \\
\hline & $\begin{array}{l}\text { Marketwired: Conferencia Impacto Digital de la Sociedad de } \\
\text { Relaciones Públicas de 2013 (PRSA) }\end{array}$ & Artículo \\
\hline & Revista: PrWeek & 2 Artículos \\
\hline & Revista digital: E-Content & Artículo \\
\hline & Revista. PrNeews & 2 Artículos \\
\hline & Revista: Public Relations Strategist .(Public Relations of America) & Artículo \\
\hline & Journal of Professional Communication & Artículo \\
\hline & Revista: PrWeek & Artículo \\
\hline & Revista: Governance Directions. (Australia) & Artículo \\
\hline & Revista: PrNews & 4 Artículos \\
\hline & Revista trimestral: The Conference Board Review & Artículo \\
\hline & Revista: Mediaskop (Rusia) & Artículo \\
\hline & Revista: Public Relations Tactics (Public Relations of America) & Artículo \\
\hline & Revista Internacional de Relaciones Públicas & Artículo \\
\hline & Revista: PrWeek & Artículo \\
\hline 2015 & Faculties of Arcadia University. Degree of Master of Arts & TFG \\
\hline & Journal of International Social Research & Artículo \\
\hline & Indian Journal of Science and Technology & Artículo \\
\hline & Journal of Wuhan Institute of Physical Education & Artículo \\
\hline & Universidad de Lund. Departamento de Comunicación Estratégica \\
\hline & The Journal (Newcastle, England). & \multicolumn{1}{|c|}{ Arto } \\
\hline
\end{tabular}




\begin{tabular}{|c|l|c|}
\hline & $\begin{array}{l}\text { Libro: Social Media Rules of Engagement: Why your online narrative is } \\
\text { the best weapon during a crisis }\end{array}$ & 1 Capítulo \\
\hline 2016 & Revista: Public Relations Strategist (Public Relations of America) & Artículo \\
\hline & Journal: Public Relations Review. & Artículo \\
\hline & Institute for Public Relations & Informe/Estudio \\
\hline & European Communication Monitor & Informe/Estudio \\
\hline & Escuela Rusa de Relaciones Públicas & Artículo \\
\hline & Revista: El profesional de la información & Artículo \\
\hline & Lunds University. Master's Programme in Management. Sweden. & TFM \\
\hline & Journal of Communication Management & Artículo \\
\hline & Revista: Nauka-Rastudent (Rusia) & Artículo \\
\hline & University of Utah. Phd Philosophy & Tesis doctoral \\
\hline 2017 & Corporate Communications: An International Journal. & Artículo \\
\hline & NZ Marketing Magazine & Artículo \\
\hline & Libro: Primer of Public Relations Research. Third Edition & 1 Capítulo \\
\hline TOTAL & \multicolumn{2}{|c}{$\mathbf{4 1}$} \\
\hline
\end{tabular}

Fuente: Elaboración propia

\section{Resultados}

Del total de 41 trabajos analizados, 32 son artículos publicados en revistas de diversas disciplinas, destacando el campo de las relaciones públicas, lo que tiene sentido dados los objetivos del estudio.

PrNews, un boletín semanal de comunicación, relaciones públicas y marketing, es la revista que más artículos publica, con un total de seis. Las dos revistas siguientes que incluyen Public Relations Tactics y Public Relations Strategist (revista trimestral), ambas de la Public Relations Society of America, incluyen cuatro artículos. La revista PrWeek, una revista de relaciones públicas y comunicación publicó tres artículos. Cabe señalar que tanto PrNews como PrWeek son revistas muy vinculadas al ámbito profesional, y no tanto al académico.

Se localizaron 12 publicaciones en revistas de diversos ámbitos profesio- nales: del área de la comunicación corporativa (Corporate communications an international journal), de marketing (NZ marketing; Marketwired) y de comunicación (El profesional de la información; Scire; Journal of communication management).

Las siete publicaciones restantes se publicaron en las siguientes revistas: tres artículos en revistas rusas (NaukaRastudent; Escuela Rusa de Relaciones Públicas y Mediaskop); un artículo en la revista del instituto de educación física de Wuhan (China); un artículo en la revista australiana (Governance Directions). Otra publicación en el Indian Journal of Science and Technology y finalmente una publicación en el Journal of Professional Communication (apoyado por el Consejo de Investigación de Ciencias Sociales y Humanidades de Canadá (SSHRC).

Otros trabajos fueron publicados como capítulos en tres libros: 
- Share this too: more social media solution for PR professionals. Collister (2013).

- Social media rules of engagement: Why you online narrative is the best weapon during a crisis. Majestic (2015).

- Primer of Public Relations Research. Stacks (2017).

Cuatro trabajos proceden del ámbito estrictamente académico siendo dos de ellos trabajos fin de grado, otro de fin de máster y una tesis doctoral. Ambos TFGs son de 2015. Uno de ellos estudia la vigilancia y la privacidad en la era digital como guía para las relaciones públicas y el segundo se trata de un estudio cualitativo de cómo se entiende Big Data en el campo de relaciones públicas El trabajo fin de master es de 2106 y estudia el impacto y potencial del Big data en Relaciones Públicas. La tesis doctoral también es de 2016 y examina cómo los individuos utilizan los medios sociales para responder a situaciones de crisis, durante y después del evento (From Crisis to Crisis: A Big Data, Antenarrative Analysis of How Social Media Users Make Meaning During and After Crisis Events. Bair, 2016).

Por último, se clasificaron dos informes/estudios que permiten obtener una visión sobre la aplicación de big data en el ámbito profesional de las relaciones públicas En primer lugar, "The Public Relations Big Data Revolution" (Institute for Public Relations, 2016), realiza un análisis de los desafíos del uso de Big Data en el campo de las relaciones públicas y como las organizaciones pueden implementarlo. Y, en segundo lugar, el informe "Exploring trends in big data, stakeholder engagement and strategic communication: Results of a survey in 43 Countries" (European Monitor, 2016), que analiza el estado actual de Big data en comunicación y en el área de las relaciones públicas.

En cuanto a los años de publicación de los trabajos, la mayor cantidad se produjo entre el 2013 al 2016. Es llamativo y precisará investigación futura la localización de un trabajo en 2005 y la ausencia en los años siguientes, así como la disminución del número de trabajos en 2017 frente a los años previos. Puede tratarse de limitaciones de las herramientas de búsqueda. Por un lado, puede ser que no estén digitalizados para su búsqueda muchos de los trabajos anteriores a 2013. Y, por otro lado, que las revistas más recientes tengan embargados sus contenidos de 2017 y no sean accesibles desde las bases de datos que trabajan UNIKA o Google Académico.

El empleo de términos en castellano e inglés también puede haber condicionado los resultados encontrados, así como el predominio de bases de datos anglosajonas. En el conjunto de 41 trabajos analizados, el idioma destacado es el inglés, ya que el 80,5\% de los autores utilizó dicha lengua para sus publicaciones. En español y en ruso se publicaron tres publicaciones, respectivamente.

\subsection{Actividades de Relaciones Públicas}

Entre las actividades más discutidas por los investigadores, destaca la comunicación interna con 26 publicaciones, relacionada de una u otra forma con la 
gestión de datos dentro de las organizaciones (Ver Gráfico 1). Diversos autores (Rockland, 2013; Roderick, 2013; Weinner \& Kochard, 2016b), apuntan que, a través de la gestión de Big Data, se puede mejorar la calidad de la comunicación entre el equipo de relaciones públicas y la dirección.
En ésta misma línea, Laing (2013) señala que Big Data es un elemento crucial de la práctica exitosa de la comunicación. Así como también, la correcta gestión de datos permite identificar y rastrear tendencias para poder adaptar las estrategias de comunicación en las organizaciones (Stephen, 2013).

Gráfico 1. Actividades de Relaciones Públicas

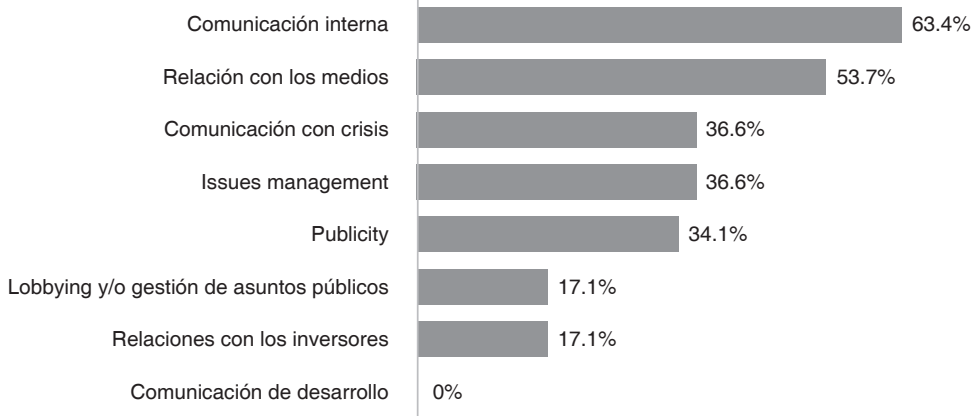

Fuente: Elaboración propia

La segunda actividad con mayor número de referencias es la relación con los medios. Los investigadores prestan especial interés cuando se trata de discutir la integración de Big Data para la monitorización de los medios, en particular los medios on-line (Gideon, 2013; Rockland, 2014). La monitorización es útil para orientar la comunicación con los públicos a través de las plataformas y redes sociales (Wichels, 2014).

Como apunta Laing (2013) en su artículo "Slow down and smell data", los profesionales de la comunicación están expuestos a los contenidos disponibles a través de los canales de medios socia- les y digitales enfrentándose a las oportunidades y desafíos de Big Data.

Respecto a la actividad de comunicación de crisis se cita en 15 de las publicaciones (36,6\% de los 41 trabajos). Aunque no es la actividad más destaca$\mathrm{da}$, se resalta la importancia de que los investigadores de crisis sean capaces de utilizar eficazmente el análisis de contenido a gran escala (Grimmer \& Stewart, 2013). La recopilación de datos permite analizar la situación actual, predecir el futuro cercano y mejorar la precisión de la toma de decisiones (Choi, 2015), siendo fundamental para el profesional de relaciones públicas cuando se trata de una crisis. 
La gestión de Big Data en comunicación de crisis está cobrando cada vez mayor interés por parte de los investigadores, debido a la gran cantidad de información existente, que en muchas ocasiones es negativa y puede conducir a una crisis comunicacional, que debe ser evitada como apunta Wichels (2014).

La actividad de issues management aparece en 15 trabajos $(36.6 \%$ de los 41 clasificados). Destaca el interés por los investigadores (Gideon, 2013; Rockland,2013) en esta labor, atendiendo al hecho de que las acciones y conversaciones se desarrollan al margen de las empresas, en un sistema abierto y democratizado, de difícil control y gestión para la reputación corporativa (Tapscott \&Williams, 2007).

Hemos vinculado la actividad de Publicity a 14 trabajos (un 34,1\% del total). Esta actividad se ve afectada particularmente por el Big data, dado que se ha producido un cambio paradigmático que requiere adaptación de estrategias tradicionales de comunicación y de relaciones públicas, a nuevos modelos de comunicación online y offline (Wichles, 2014). Dentro de esta misma línea, Choi (2015), apunta que los datos no estructurados se generan en grandes cantidades y es ahí donde se puede tanto inferir como captar la opinión pública.

La gestión de asuntos públicos se aborda en siete publicaciones $(17,1 \%$ del total). Autores como Choi (2015) consideran que es necesario desarrollar un nuevo tipo de paradigma de relaciones públicas cuando se trata de asuntos de política pública.
La actividad de relaciones con los inversores se desarrolla en un total de siete publicaciones (un 17,1\% del total) $\mathrm{Si}$ bien no es la actividad más desarrollada, el nuevo entorno de comunicación 3.0. obliga a que sea de interés para los profesionales de relaciones públicas por las oportunidades que se presentan en la gestión de datos para ser un intermediario entre las partes interesadas y el equipo externo de comunicaciones (Roderick, 2014).

Finalmente, la actividad de comunicación de desarrollo no se discute en ninguno de los trabajos. Será interesante ver si se trabaja más el tema en el futuro y/o en trabajos no recogidos en las bases de datos utilizadas en la búsqueda.

\subsection{Temáticas desarrolladas}

Entre las temáticas de los trabajos analizados, son de especial interés las vinculadas a los retos y oportunidades que ofrece Big Data a los profesionales de Relaciones públicas (Houltzhausen, 2016; Stacks, 2017; Weiner \& Kochhar, 2016), tanto en las organizaciones, como en las propias agencias de relaciones públicas (Mcmahon, 2015; Martin, 2014; Roderick, 2014; Swinson \& Woliansky 2014; Waddington, 2013).

La gestión de datos en tiempo real es otro tema que se considera fundamental para desempeñar la labor de Relaciones Públicas (Johnson, 2013; Weiner \& Kochhar, 2016), debido a las implicaciones que tiene una gestión rápida y oportuna de la información tanto como para resolver crisis o establecer un diálogo directo con los stakeholders. 
La integración de Big Data, el análisis inteligente del mismo y cómo el análisis predictivo puede agregar valor a la medición (Rockland, 2014), son otros temas desarrollados en los trabajos publicados. Dentro de la gestión de datos, la descripción de cómo utilizar y administrar herramientas que faciliten esta labor también es otra temática elegida por los investigadores (Terentyeva, Morbakh \& Vozgrina, 2016; Van der Meer, 2016; Weiner \& Kochhar, 2016).

El área de la privacidad (McMahon, 2015; Swinson \& Woliansky, 2014) y los problemas legales, en cuanto exposición de datos (Holtzhausen, 2016; Swinson \& Woliansky, 2014) son también objetivo de estudio, y de gran relevancia al utilizar Big data. La protección de datos es un tema de interés general que cobra particular significación para la disciplina de Relaciones Públicas debido a la constante relación que se tiene con la información de diversos públicos de interés de las organizaciones.

Finalmente, el estado actual de la investigación sobre Big Data y comunicación (Choi, 2015; Kajander \& Sivard 2015; Swinson \& Woliansky 2014; Wiencierz \& Röttger, 2017) y el cambio de paradigma comunicacional que trae consigo la gestión de Big data (Cobertt \& Docherty, 2016) son cuestiones desarrolladas por los autores, aunque no son los principales temas de discusión en los trabajos clasificados.

\section{Discusión y conclusiones}

El objetivo de la presente publicación fue conocer el estado actual de la inves- tigación en la gestión de Big data en relaciones públicas. La búsqueda de trabajos se realizó durante el mes de diciembre de 2017, localizando 41 trabajos relacionados con el objeto de estudio.

Consideramos que la cantidad de trabajos que abordan explícita o implícitamente la gestión de Big Data en relaciones públicas es escasa, tomando en cuenta que trabajar con datos masivos puede ayudar a "administrar las relaciones públicas de manera más estratégica” (Wiencierz \& Röttger, 2017: 264). Así lo reconocen también trabajos previos, que señalan la necesidad de ahondar más en el tema del potencial del Big Data para la ciencia de la comunicación (Chen, et al, 2012; Parks, 2014; Wiencierz \& Röttger, 2017), y para los profesionales de relaciones públicas.

Es importante no quedar atrás respecto a disciplinas como el marketing o la publicidad, sectores pioneros en valorar la importancia de Big Data (Bustamante \& Guillén 2017). Si la tendencia continua y los profesionales de relaciones públicas no se dan cuenta del potencial de la gestión de datos, se corre el riesgo de quedar muy por detrás de otros campos (Kent \& Saffer, 2014), ignorando el potencial de las contribuciones de la disciplina de relaciones públicas al éxito del sistema Big Data en las organizaciones (Yanik \& Göztaş, 2015).

Los estudios encontrados en la investigación, realizados por la European Public Relations Education and Research Association (Euprera) y la European Association of Communication Directors 
EACD (2016) también confirman esta situación. Según sus datos, un 59,3\% de los profesionales de relaciones públicas asegura prestar suficiente atención al tema, y tan solo un $21,2 \%$ ha implementado Big Data a sus actividades. Tales porcentajes revelan la necesidad de comenzar a trabajar en el área.

Las publicaciones analizadas en este trabajo muestran que las actividades de comunicación interna, relaciones con los medios, comunicación de crisis e issues management son las más vinculadas a los datos masivos.

En el campo de la comunicación interna podemos estudiar cómo la gestión de datos puede afectar en la creación o generación de conocimiento a partir de la administración de información de calidad, y como a través de ella se puede contribuir al éxito en el cambio de mentalidad de las organizacio- nes, hacia la transformación digital (Klewes, et al, 2017).

En las áreas de relaciones con los medios, comunicación de crisis e issues management, el big data gana peso dada la enorme cantidad de datos que se producen en la red. Este diálogo digital necesita ser atendido, no solo por las implicaciones éticas de recopilar y usar toda esta información extraída de diversos medios, sino también, por la necesidad de un cambio en la relación de las organizaciones con todos sus públicos.

El Big data "enfrentará a expertos de todo tipo con el desafío fundamental de crear valor a partir de aplicaciones basadas en datos" (Klewes, et al, 2017: 3). La correcta aplicación de Big Data en las actividades de Relaciones Públicas y su estudio en el ámbito académico contribuirán, al éxito de las estrategias de comunicación basadas en datos.

\section{Bibliografía}

Benítez-Eyzaguirre, L. (2016). Análisis de la recomendación entre iguales en la reputación online de las organizaciones. El profesional de la información, 25(4), 652-660. http://dx.doi. org/10.3145/epi.2016.jul.15

Beyer, M. \& Laney, D. (2012). The Importance of 'Big Data: A Definition. Gartner, G00235055.

Boyd, D. \& Crawford, K. (2012). Critical questions for Big Data: Provocations for a cultural, technological, and scholarly phenomenon. Information, Communication y Society, 15(5), 662679. doi: 10.1080/1369118X.2012.678878

Bustamante, A., B., y Guillén A., S. (2017). Un acercamiento al Big Data y su utilización en comunicación. Mediaciones Sociales, 16, 115-134. http://dx.doi.org/10.5209/MESO.58112

Broom, G. y Sha, B.L. (2013). Effective Public Relations (9a.ed.). Boston: Pearson. Cutlip and Center's. Cobertt, H. \& Docherty, J. (2016). Managing Change in the Public Relations Industry: The Impact and Potential of Big Data. (Master Thesis).

Cutlip, S., Center, A. \& Broom, G. (2001). Relaciones Públicas eficaces. Barcelona: Gestión 2000.

Chartred Institute of Public Relations (CIPR) edited Waddington, S. (2013). Share This Too: More Social Media Solution for PR Professionals. Jhon Wiley \& Sons.

Chen, H., Chiang, R. \& Storey, V. (2012). Business intelligence and analytics: From big data 
to big impact. MIS Quarterly, 36(4), 1165-1188.

Choi, M.W. (2015). The Customized Public Policy PR Strategy in Big Data Era. Indian Journal of Science and Technology, 8(23), 1-8.

Davenport, T. H., Barth, P. \& Bean, R. (2012). "How 'Big Data' is different” MIT Sloan Management Review. Recuperado de https://sloanreview.mit.edu/article/how-big-data-is-different/

Gandomi, A. \& Haider, M. (2015). Beyond the hype: Big data concepts, methods, and analytics. International Journal of Information Management, 35(2), 137-144. https://doi. org/10.1016/j.ijinfomgt.2014.10.007

Gartner Research (2012). Recuperado de https://www.gartner.com/doc/2100215?ref=mrktg-srch

George, G., Haas, M.R. \& Pentland, A. (2014). Big data Management. Academy of Management Journal. 57(2), 321-326.

Gideon, F. (2013). Useful information. PrWeek. 16(10), 38-42. Recuperado de https://www. prweek.com/article/1274489/measurement-roundtable-useful-information,

Grimmer, J. \& Stewart, B.M. (2013). Text as data: The promise and pitfalls of automatic content analysis methods for political texts. Political Analysis, 21(3), 267-297. doi:10.1093/pan/mps028

Holtzhausen, D. (2016). Datafication: threat or opportunity for communication in the public sphere? Journal of Communication Management, 20(1), 21-36. https:/doi.org/10.1108/ JCOM-12-2014-0082

Johnson, D. (2013). Big Data = Big Business: Put Social Media Analytics Into Action.

Marketwired. Recuperado de http://www.marketwired.com/press-release/

big-data-big-business-put-social-media-analytics-into-action-presented-marketwired-1806309.

Kajander, L. \& Sivard, E. (2015). Size matters? En kvalitativ studie kring hur bid data förstås inom fältet PR. Recuperado de https://www.lunduniversity.lu.se/lup/publication/5463867

Kent, M \& Saffer, A. (2014). A Delphi study of the future of new technology research in public relations. Public Relations Review, 40, 568-576. https://doi.org/10.1016/j. pubrev.2014.02.008

Kerlinger, F. N. \& Lee, H. B. (2000). Foundations of behavioral research (4th ed.). New York: Holt, Rinehart \& Winston.

Klewes, J.; Popp, D. \& Rost-Hein, M. (2017). Out-thinking Organizational Communications: The Impact of Digital Transformation. Switzerland: Springer.

Laing, A. (2013). Slow down and smell Data. Journal of Professional Communication, 3(1), 197-200.

Martin, D. (2014). Mining the future of PR. The Conference Board Review, 82-83.

Martin, I. (2007). Retos de la comunicación corporativa en la sociedad del conocimiento: de la gestión de información a la creación de conocimiento organizacional. Signo y Pensamiento, 51(XXV), 52-67. Recuperado de http://www.redalyc.org/articulo.oa?id=86005106

Mcmahon, R. (2015). Surveillance and Privacy in the Digital Age: A Primer for Public Relations. Graduate Theses. Pensilvania. Arcadia Universty. Recuperado de Scholars Work Arcadia.

Orozco, J. A. y Ferre, C. (2012). Los índices de reputación corporativa y su aplicación en las empresas de comunicación. III Congreso Asociación Española de Investigación de la Comunicación. Tarragona, 1-19. Recuperado de http://www.aeic2012tarragona.org. 
Parks, M. (2014). Big data in communication research: its contents and discontents. Journal of Communication, 64(2), 355-360. https://doi.org/10.1111/JCOM.12090

Puyol, J. (2015). Aproximación jurídica y económica al Big Data. Valencia: Tirant Lo Blanch.

Rockland, D. (2014). Integration: a look ahead to 2015. Public Relations Tactics, $21,7$. Recuperado de https://www.apps.prsa.org

Roderick, J. (2013). How to demystify the power of Big Data. PrNews. Recuperado de http:// www.prnewsonline.com/how-to-demystify-the-power-of-big-data/

Shahin, S. (2016). A Critical Axiology for Big Data Studies. Palabra Clave, 19(4), 972-996. doi: 10.5294/pacla.2016.19.4.2

Shilina, M. \& Levchenko, V. (2014). Big Data, Open Data, Linked Data, Metadata in PR: Current models in the transformation of theory and practice. Mediaskop, 1, 16-16. Recuperado de http://www.mediascope.ru/en/big-data-open-data-linked-data.

Stacks, D. W. (2017). Primer of Public Relations Research. Third edition. New York: The Guilford press.

Swinson, M. \& Woliansky, A. (2014). Big data is coming and it's getting personal. Governance Directions, 66, 340-344. Recuperado de https://search.informit.com.au/documentSummary; $\mathrm{dn}=407960089332896$; res $=$ IELAPA $>$ ISSN : 2203-4749.

Tapscott, D. \& Williams, A. (2007). Wikinomick: la nueva economía de las multitudes inteligentes. Barcelona: Paídos.

Terentyeva E.I., Morbakh E.S. \& Vozgrina A.V. (2016). Methods of Using Big Data for PRtasks. Nauka-Rastudent. 3(27). http://nauka-rastudent.ru/27/3333/

Van Der Meer, T. (2016). Automated content analysis and crisis communication research. Public Relations Review,42, 952-961. https://doi.org/10.1016/j.pubrev.2016.09.001

Wang, H.; Xu, Z.; Fujita, H. \& Liu, S. (2016). Towards felicitous decision making: An overview on challenges and trends of Big Data. Information Sciences, 1-19. https://doi.org/10.1016/j. ins.2016.07.007

Weinner, M. \& Kochhar, S. (2016a). Irreversible: The Public Relations Big Data Revolution. IPR Measurement Commission. Recuperado de http:/www.instituteforpr.org/wpcontent/ uploads/IPR_PR-Big-Data-Revolution_3-29.pdf

Weinner, M. \& Kochhar, S. (2016b). On the Horizon. Unleashing the Power of Big Data in Public Relations. Public Relations Strategist, 22, 12-13. Recuperado de http://www.apps.prsa.org

Wiencierz, C. \& Röttger, U. (2017). The use of big data in corporate communication. Corporate Communications. An International Journal, 22(3), 258-272. https:/ doi.org/10.1108/ CCIJ-02-2016-0015

Wichels, S. (2014). Nuevos desafíos en Relaciones Públicas 2.0. La creciente influencia de las plataformas de online review en Turismo. Revista internacional de relaciones públicas, 7(4), 197-216. http://hdl.handle.net/10630/7928

Xifra, J. (2014). Manual de relaciones públicas e institucionales (2a. ed.). Madrid: Tecnos.

Yanik, A. \& Göztaş, A. (2015). Big data systems, business intelligence and public relations. Journal of International Social Research, 8, 822-826. 